\title{
The Effect of Photoacclimation on Photosynthetic Energy Storage Efficiency, Determined by Photoacoustics
}

\author{
Yulia Pinchasov-Grinblat ${ }^{*}$, Razy Hoffman, Zvy Dubinsky \\ Faculty of Life Sciences, Bar-Ilan University, Ramat-Gan, Israel \\ E-mail: yulia.pinchasov@gmail.com \\ Received May 11, 2011; revised May 23, 2011; accepted June 8, 2011
}

\begin{abstract}
Photosynthesis rates in phytoplankton depend on light intensity and its spectral composition, however their relation changes with photoacclimation. During the photoacclimation process algal cells optimize their harvesting and utilization of available light through series of related physical, biophysical, biochemical and physiological changes. These changes result in the ability of phytoplankton to survive under dim light when transported to the depth of the water column and avoid photodynamic damage when exposed to the intense radiation at the surface. Any reduction in the efficiency of light utilization results in decreased rates of photosynthesis rate and slow growth. We present here the study of changes in photosynthetic energy storage efficiency of three phytoplankton species upon photoacclimation to low and high light, as measured by photoacoustics. Our results illustrate the power of photoacoustics as a tool in aquatic ecology and in the physiological research of phytoplankton.
\end{abstract}

Keywords: Phytoplankton, Photoacclimation, Photosynthesis, Photoacoustics

\section{Introduction}

Phytoplankton, like all photosynthetic organisms, depend on the capture of light and the transformation of its energy into stored photosynthate, which subsequently fuels all ecosystem activities. For the first step of the photosynthetic process, light has to be intercepted by a variety of light absorbing substances, the photosynthetic pigments. These pigments are associated with proteins, forming light harvesting arrays, or "antennae", which collect excitation energy and transfer it to the reaction centers of the two photosystems, PSI and PSII that together constitute the photosynthetic unit. These photosynthetic units have a given probability or cross-section for absorbing impinging light photons.

Because of the importance of photoacclimation for phytoplankton which is frequently transported over two orders of magnitude changes in light intensity within hours, this process received considerable attention in the study of aquatic primary production [1-3]. In all the numerous studies of the mechanisms of the photoacclimation process in phytoplankton, a common trend of increase in chlorophyll a and in other light harvesting pigments as growth irradiance decreases was observed. Photoacclimation also affects pigment ratios of phyto- plankton, as light harvesting pigments such as the chlorophylls, phycobilins, fucoxanthin and peridinin increase under low light, whereas photoprotective carotenoids like $\beta$ carothene, astaxanthin and xanthophyll cycle pigments show the opposite relation with ambient light, increasing whenever exposed to potentially harmfully high intense light [4-7]. Most photosynthetic pigments other than chloropyll $a$ are an integral part of the light harvesting antennae. These photosynthetic pigments respond to changes in light intensity in a similar way to that of chlorophyll $a$ [8]. Concomitantly, as cellular pigmentation increases in the course of photoacclimation to low light, the cross section of phytoplankton invariably decreases $[8,9]$. This change in cross section resulting from the increase in mutual shading between cellular light harvesting entities, as their density increases. It happens on all scales, among individual pigment molecules, thylakoids, chloroplasts within cells, and among cells in culture [10]. Upon exposure to high light intensity, the above described trends are reversed [11].

The photoacoustic method, allows the direct determination of the energy storage efficiency of photosynthesis by relating the energy stored by photosynthesis to the total light energy absorbed by the plant material [12-14]. Depending on the efficiency of the photosynthetic sys- 
tem, a variable fraction of the absorbed light energy is stored, thereby affecting the heat evolved and the resulting photoacoustic signal.

By exposing the cells to a saturating continuous background light, no storage of any of the pulse energy can take place, whereas in the absence of such light, a maximal fraction of the pulse energy is stored by photosynthesis. Thus the maximal efficiency $\Phi_{\max }$, is determined as the complement of the ratios of the photoacoustic signal, generated by a weak pulse of light in the dark (PAdark), to that obtained under strong continuous light (PAlight).

$$
\Phi_{\text {max }}=1-\text { PA dark } \div \text { PA light }
$$

The aim of this work was to examine the photoacoustic method developed by us [15-17] in physiological phytoplankton research. We demonstrate this novel application, by investigating changes in photosynthetic energy storage efficiency occurring in a few phytoplankton species of different taxa, resulting from photoacclimation.

\section{Methods and Materials}

\subsection{Algal Culture}

The three species of marine phytoplankton studied were a diatom, Phaeodactylum tricornutum, green alga Nannochloropsis sp. and golden-brown flagellate Isochrysis galbana. All cultures were grown in $250 \mathrm{~mL}$ Erlenmeyers flasks containing $200 \mathrm{~mL}$ enriched artificial seawater medium (Guillard's F/2) [18] at $24^{\circ} \pm 0.5 \mathrm{C}$, under white fluorescent lights at $\sim 10 \mu \mathrm{mol} \mathrm{q} \mathrm{m-2} \mathrm{s-1} \mathrm{(low} \mathrm{light} \mathrm{(LL))}$ and $\sim 500 \mu \mathrm{mol} \mathrm{q} \mathrm{m-2} \mathrm{s-1} \mathrm{(high} \mathrm{light} \mathrm{(HL)).}$

Cell concentration was measured with a hemacytometer. Chlorophyll content was measured spectrophotometrically in $90 \%$ acetone extracts using the equations of Jeffrey and Humphrey [19].

\subsection{Photoacoustics}

The experimental system is shown schematically in Figure 1. The sample was placed in a $16 \mathrm{~mm}$ square glass cell (PAC). The laser (L) pulse, after passing through a pair of $1 \mathrm{~mm}$ wide slits $(\mathrm{S})$ is incident upon the suspension of algae whose pigments absorb part of the laser light. Depending on the experimental conditions, a fraction of the absorbed light pulse is stored in the products of photosynthesis. The remainder of the absorbed light is converted to heat producing an acoustic wave. This is intercepted by a submersible detector (D), containing the ceramic disc. A small portion of the laser pulse is deflected by a beam splitter (BS) and used to trigger the Tektronix TDS 430A oscilloscope, where the amplified
(Amptek A-250 Preamp and Stanford Research A 560 Amp) photoacoustic signal is recorded. The signal contains a noisy background and later reflections from the walls of the vessel as well as from impedance mismatch within the detector (for details see [17]).

By increasing the continuous background light intensity from zero to saturation of photosynthesis, an increasing fraction of the reaction centers is closed at any time, and a decreasing fraction of the probe laser pulse energy is stored. A corresponding increase in the fraction of the pulse energy is converted to heat, which is sensed by the photoacoustic detector. From these detector responses the photosynthetic energy-storage versus background light-intensity relationship was obtained [15-17].

\section{Results and Discussion}

In these experiments the photoacclimation of three algal species Phaeodactylum tricornutum, Nannochloropsis sp and Isochrysis galbana to low and high photon irradiances was examined. In general, photoacclimation to low light results in increased cellular absorption due to high concentration of light-harvesting pigments. In the numerous studies on the mechanism of photoacclimation in phytoplankton, a common trend was reported of increase in chlorophyll as growth irradiance decreases (Falkowski, 1980; 1984; Dubinsky et al. 1986; 1995, Ritz, et al. 2000). As seen in Figure 2 and Figure 3 all three species showed a difference in cellular chlorophyll content by $\sim 56 \%$ in Isochrysis galbana, 38\% and 35\% in Phaeodactilum tricornutum and Nannochloropsis sp, respectively.

The ratios of cellular chlorophyll under the low and high light were 1.415 for Phaeodactilum tricornutum, 1.398 for Nannochloropsis sp. and 1.984 for Isochrysis

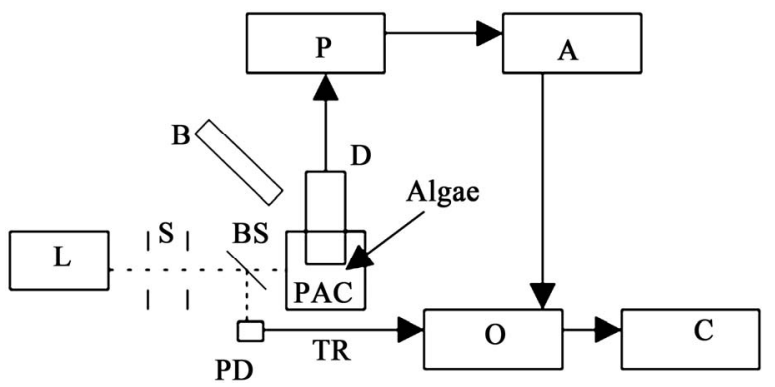

Figure 1. Schematic of the photoacoustic setup: L: laser (Minilite Q Switched Nd :YAG, 532 nm); S: beam shaping slits, BS: beam splitter; PAC: photoacoustic cell with suspension of algae; D: stainless-steel photoacoustic detector, contained a 10-mm diameter resonating ceramic disc (BM 500, Sensor, Ontario, Canada); P: low-noise preamplifier (Amptek A-250); A: low noise amplifier (SRS 560); PD: photodiode; TR: trigger signal, $B$ : background light source, quartz-halogen illuminator (Cole Parmer 4971); O: oscilloscope (Tektronix TDS 430A); C: computer. 
galbana. In addition to the changes in cellular chlorophyll most other plant pigments also respond to ambient irradiance [4,23]. All light-harvesting pigments increase under low light. These include the carotenoids fucoxantin and peridinin, in addition to all chlorolhylls, phycoerythrin and phycocyanin [4].

These results are similar to the data cited in the literature, where cellular chlorophyll content increased under low light (LL) conditions and reduced under high light (HL) (Table 1)
The decrease of chlorophyll concentration in HL growth conditions resulted in a parallel reduce in photosynthetic energy storage efficiency Figure 4.

We determined the photosynthetic energy storage under different ambient irradiance levels, resulting in an energy storage curve (Figure 5). This relationship is similar to the photosynthesis versus irradiance, (P vs I) curve obtained by the tedious standard measurements of ${ }^{14} \mathrm{C}$ fixation and oxygen evolution [24-26] or the indirect results from measurement of variable fluorescence [27].

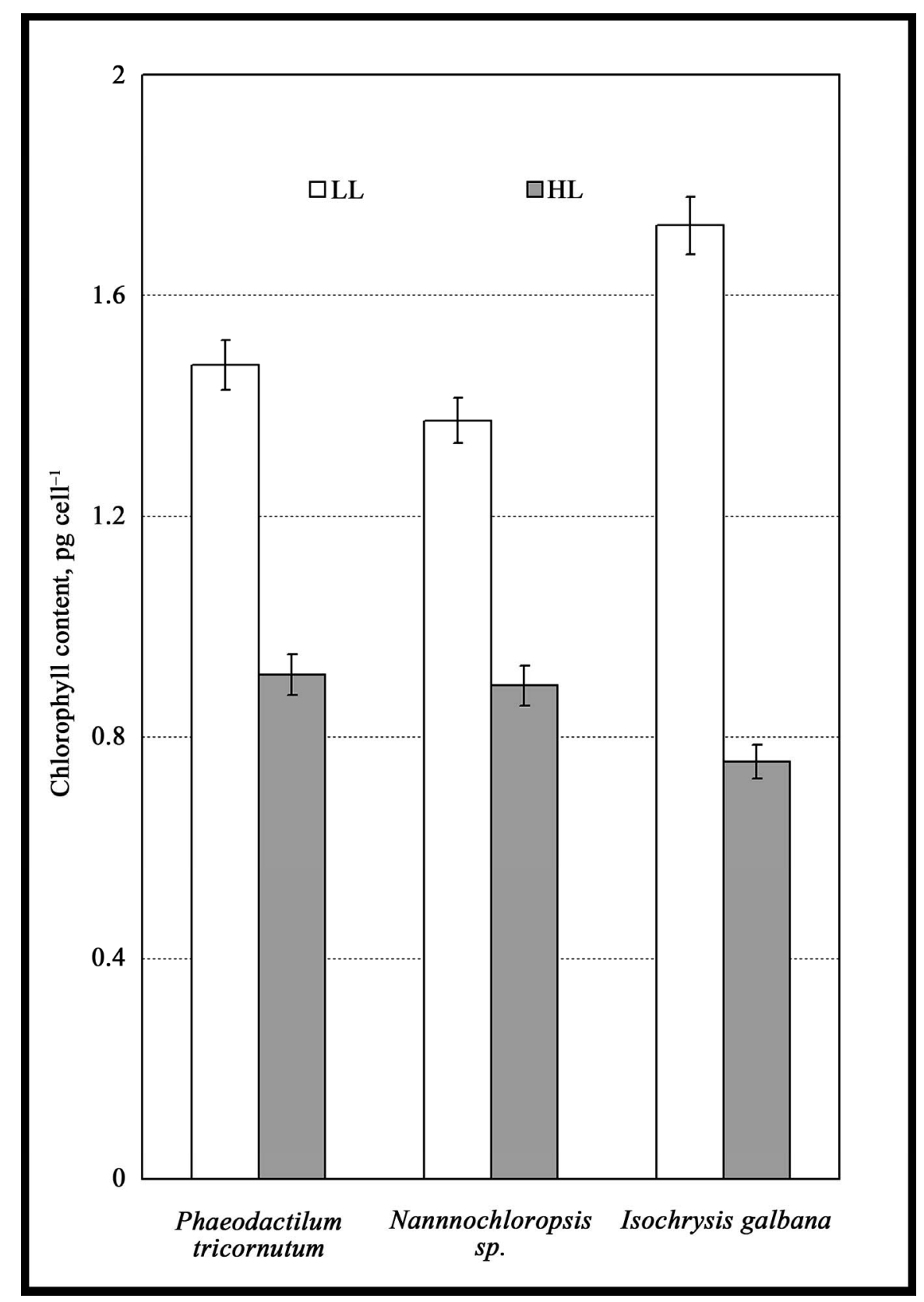

Figure 2. The effect of photoacclimation to high light $\left(500 \mu \mathrm{mol} \cdot \mathrm{q} \cdot \mathrm{m}^{-2} \cdot \mathrm{s}^{-1}\right)$ and low light $\left(10 \mu \mathrm{mol} \cdot \mathrm{q} \cdot \mathrm{m}^{-2} \cdot \mathrm{s}^{-1}\right)$ on cellular chlorophyll content for three algae. 


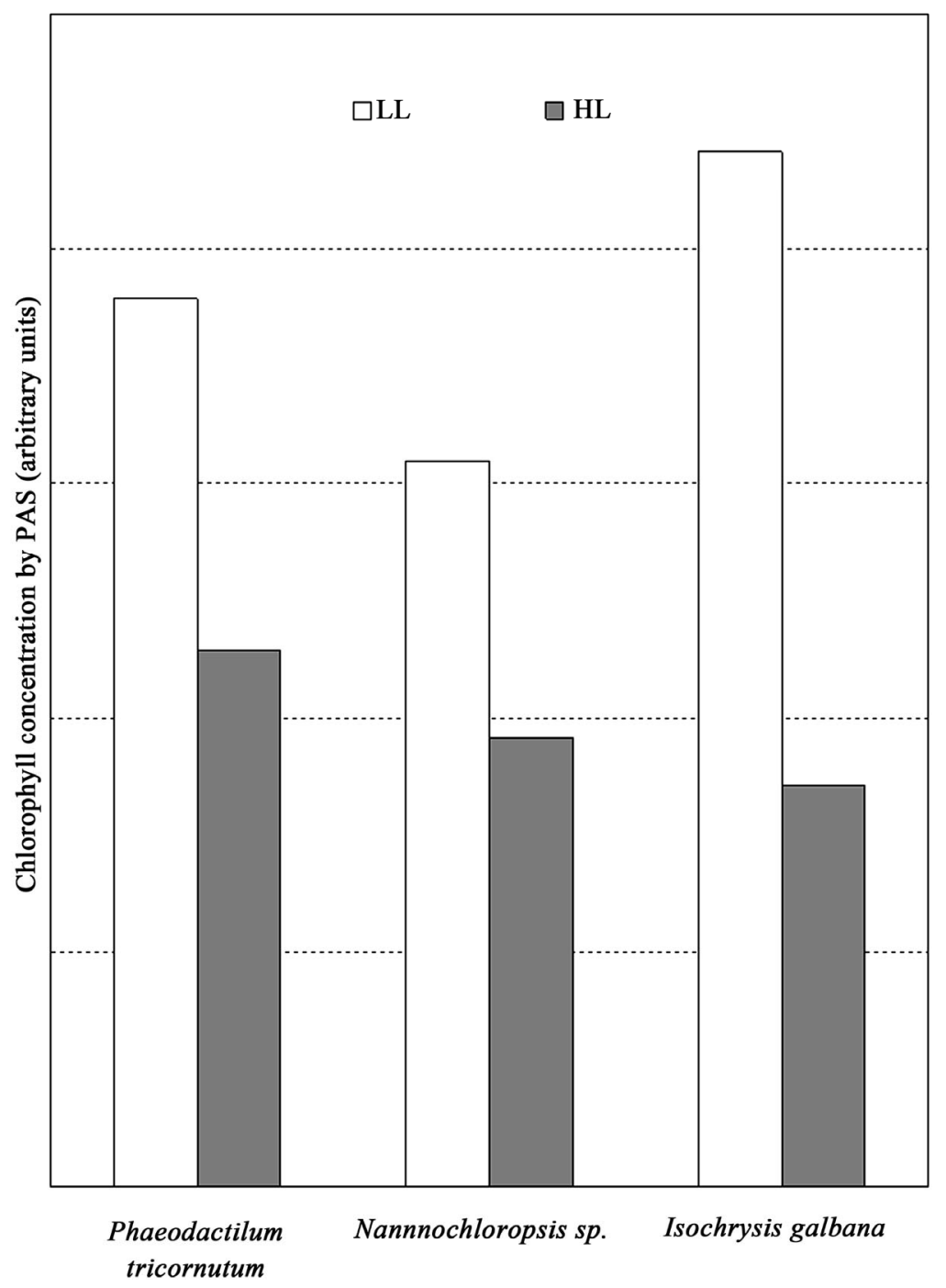

Figure 3. The effect of photoacclimation to high light and low light on chlorophyll concentration measured by photoacoustics.

Table 1. The LL/HL chlorophyll ratios for different phytoplankton species cited in literature.

\begin{tabular}{lll}
\hline \multicolumn{1}{c}{ Algae } & & \multicolumn{1}{c}{ Reference } \\
\hline Phaeodactilum tricornutum & 1.415 & Present study \\
Nannochloropsis sp. & 1.398 & Present study \\
Isochrysis galbana & 1.984 & Present study \\
Phaeodactilum tricornutum & 1.592 & Present study by photoacoustics \\
Nannochloropsis sp. & 1.020 & Present study by photoacoustics \\
Isochrysis galbana & 2.501 & Present study by photoacoustics \\
Thalassiosira weisflogii & 2.486 & Dubinsky et al. 1986 \\
Isochrysis galbana & 2.130 & Dubinsky et al. 1986 \\
Prorocentrum micans & 2.351 & Dubinsky et al. 1986 \\
Symbiodinium microadriaticum & 1.921 & Iglesias Prieto and Trench, 1994 \\
Isochrysis galbana & 2.076 & Herzig and Dubinsky, 1992 \\
\hline
\end{tabular}




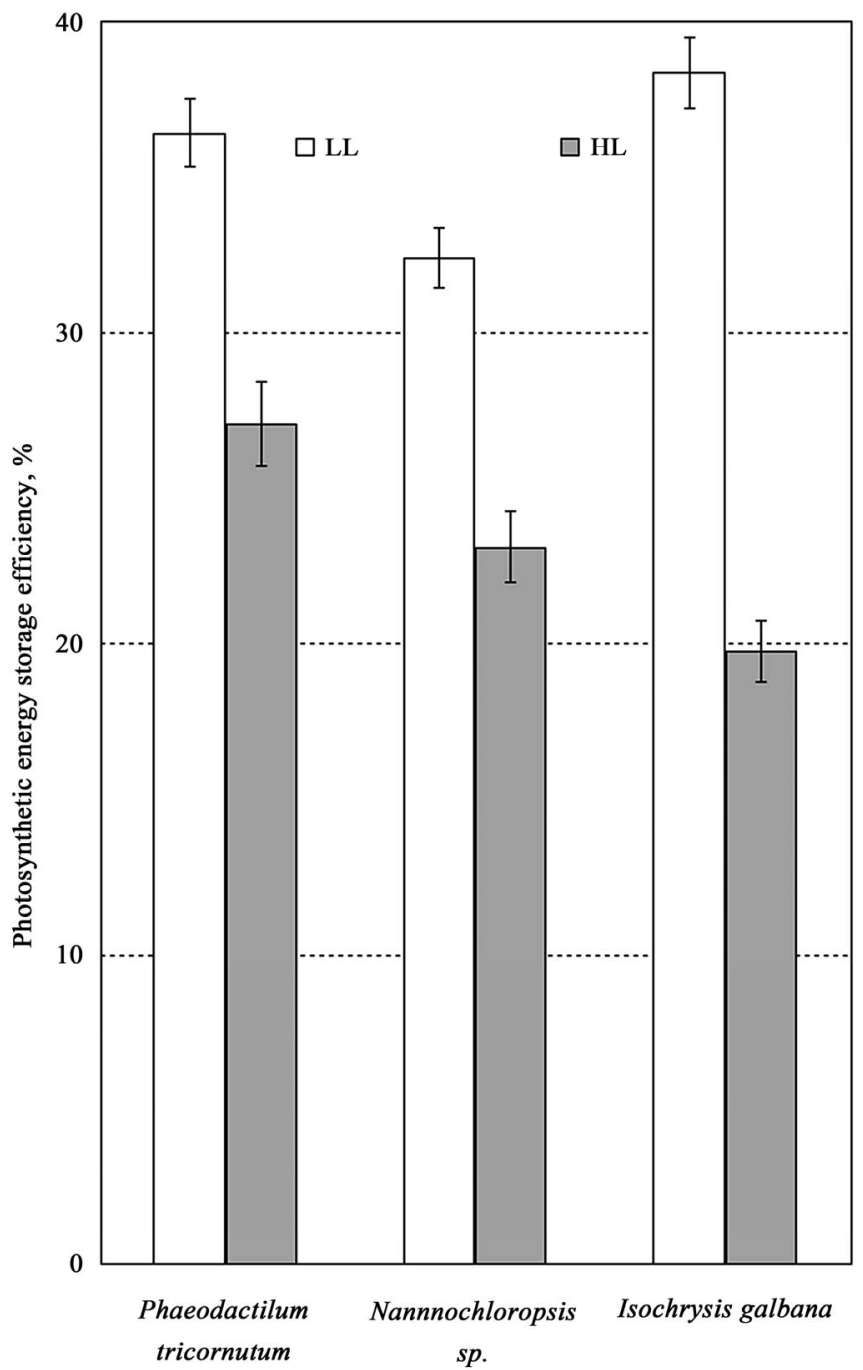

Figure 4. The effect of photoacclimation to high light and low light on photosynthetic energy storage efficiency for three algae.

Figure 5 shows that the photosynthetic energy storage efficiency Isochrysis galbana at the initial time of experiments resembles the usual $\mathrm{P}$ vs I curves, where photosynthesis increases with light intensity up to the onset of light saturation. Thus, neither the initial slope, $\alpha$, nor the light saturation parameter $E_{k}$, change. These photosynthetic parameters relate to the efficiency of light harvesting by the pigments which seems to remain unaffected. The effect of photoacclimation to high light is however pronounced in the depression of the light saturated rate of photosynthesis $\mathrm{P}_{\max }$. After the exposure to HL, all samples shows decrease in photosynthetic energy storage efficiency by $48 \%$ in Isochrysis galbana, $29 \%$ and $26 \%$ in Phaeodactilum tricornutum and Nannochloropsis $s p$., respectively.

The results of this study are similar to those carried out in many previous studies $[8,21,22,28]$. However, these studies used a variety of measures of the photo- synthetic activity and are thus difficult to compare directly. Many also involved difficult and labor intensive measurements. In contrast, our measurements were all carried out with the same methodology that is simple, quick and direct. The complete measure of activity requires only one minute. Most important, the activity measured by the photoacoustic method is the absolute thermodynamic efficiency of photosynthesis. Therefore, while there is ample evidence that under acclimation to high light there is a universal decrease in cellular pigmentation, photoacoustic reveals what fraction of the absorbed light is dissipated as heat. Indeed, under high light acclimation there is an increase in photoprotective pigments that do not make the light they absorb available for photochemistry but rather dissipate it as heat. Such are some of the carotenoids like $\beta$ carotene and astaxanthine as well as the pigments involved in the xanythophyll cycle. 


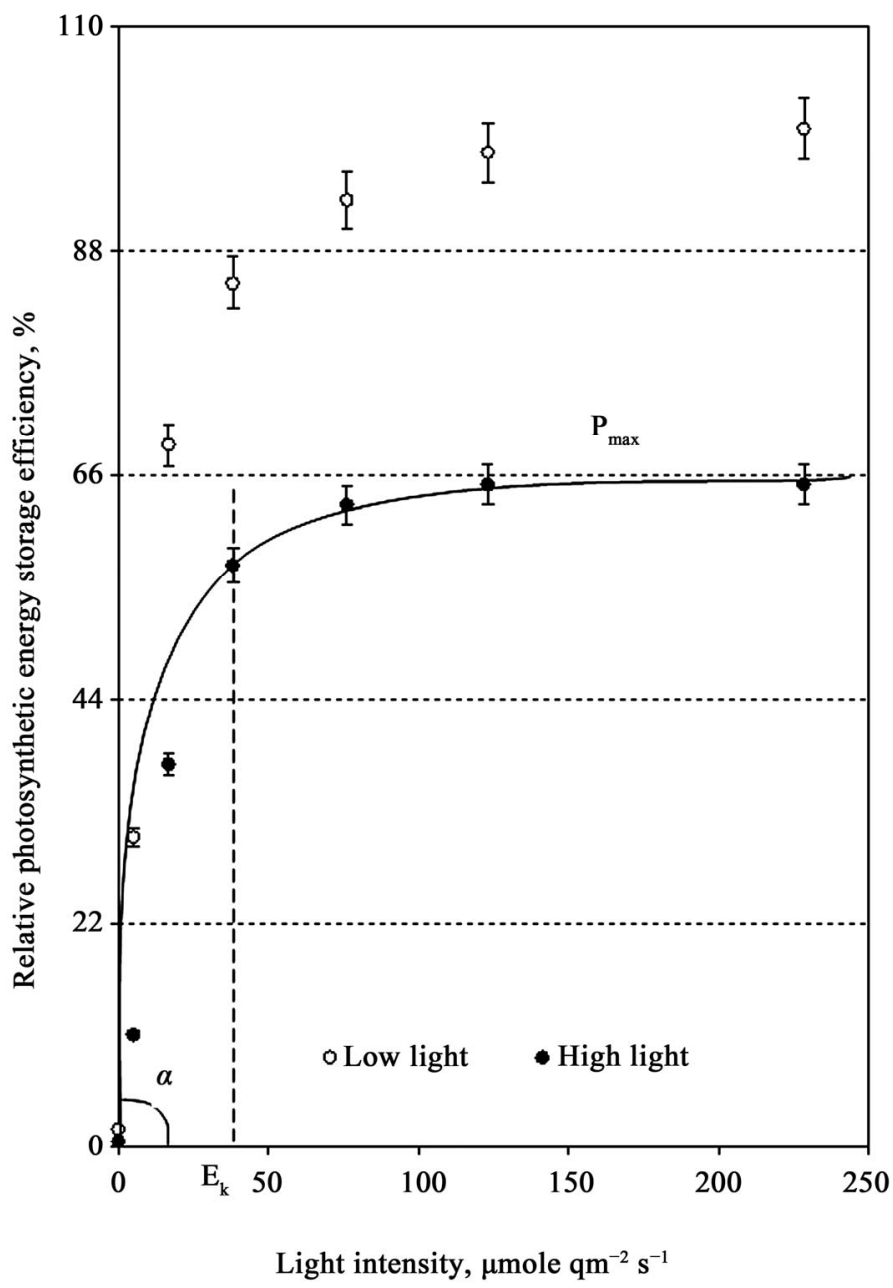

Figure 5. The effect of photoacclimation to high light and low light on relative photosynthetic energy storage efficiency of Isochrysis galbana. In order to standardize our results, we converted the absolute energy storage efficiencies to relative ones, setting the maximal storage in low light conditions as $\mathbf{1 0 0} \%$ photosynthetic energy storage efficiency.

Furthermore, reliable estimates of the quantum yields of oceanic phytoplankton are essential parameters for any modeling of global primary productivity, based on interpretation of satellite images. In summary it is both of considerable scientific interest, and of great applied importance for fisheries and for environmental protection to measure changes over time, as well as over regional variation, of the efficiency of photosynthetic energy storage.

\section{References}

[1] P. G. Falkowski, "Physiological Response of Phytoplankton to Natural Light Regimes," Journal of Plankton Research, Vol. 6, No. 2, 1984, pp. 295-307. doi:10.1093/plankt/6.2.295

[2] P. G. Falkowski and J. LaRoche, "Acclimation to Spectral Irradiance in Algae," Journal of Phycology, Vol. 27, No. 1, 1991, pp. 8-14. doi:10.1111/j.0022-3646.1991.00008.x
[3] Z. Dubinsky, "Light Utilization Efficiency in Natural Phytoplankton Communities," In: P. G. Falkowski, ed., Primary Productivity in the Sea, Plenum Press, New York, 1980, pp. 83-97.

[4] Z. Dubinsky and N. Stambler, "Photoacclimation Processes in Phytoplankton: Mechanisms, Consequences, and Applications," Aquatic Microbial Ecology, Vol. 56, No. 2-3, 2009, pp. 163-176. doi:10.3354/ame01345

[5] M. Schagerl and B. Muller, "Acclimation of chlorophyll a and carotenoid levels to different irradiances in four freshwater cyanobacteria," Journal of Plant Physiology, Vol. 163, No. 7, 2006, pp. 709-716. doi:10.1016/j.jplph.2005.09.015

[6] M. C. Wang, D. Bohmann and H. Jasper, "JNK Signaling Confers Tolerance to Oxidative Stress and Extends Lifespan in Drosophila," Developmental Cell, Vol. 5, No. 5, 2003, pp. 811-816. doi:10.1016/S1534-5807(03)00323-X

[7] C. M. Fan, J. A. Porter, C. Chiang, D. T. Chang, P. A. Beachy and M. Tessierlavigne, "Long-Range Sclerotome Induction by Sonic Hedgehog-Direct Role of the 
Amino-Terminal Cleavage Product and Modulation by the Cyclic-AMP Signaling Pathway," Cell, Vol. 81, No. 3, 1995, pp. 457-465. doi:10.1016/0092-8674(95)90398-4

[8] Z. Dubinsky, R.Matsukawa and I. Karube, "Photobiological Aspects of Algal Mass Culture," Journal of Marine Biotechnology, Vol. 2, 1995, pp. 61-65.

[9] Z. Dubinsky, P. G. Falkowski and K. Wyman, "Light Harvesting and Utilization in Phytoplankton," Plant \& Cell Physiology, Vol. 27, No. 7, 1986, pp. 1335-1350.

[10] T. Berner, K. Wyman, Z. Dubinsky and P. G. Falkowski, "Photoadaptation and the "Package Effect" in Dunaliella Tertiolecta (Chlorophyceace)," Journal of Phycology, Vol. 25, No. 1, 1989, pp. 70-81. doi:10.1111/j.0022-3646.1989.00070.x

[11] A. F. Post, Z. Dubinsky, K. Wuman and P. G. Falkowski, "Kinetics of Light-Intensity Adaptation in a Marine Planktonic Diatom," Marine Biology, Vol. 83, No. 3, 1984, pp. 231-238. doi:10.1007/BF00397454

[12] S. Malkin and D. Cahen, "Photoacoustic Spectroscopy and Radiant Energy Conversion: Theory of the Effect with Special Emphasis on Photosynthesis," Photochemistry and Photobiology, Vol. 29, No. 4, 1979, pp. 803-813. doi:10.1111/j.1751-1097.1979.tb07770.x

[13] O. Canaani, S. Malkin and D. Mauzerall, "Pulsed Photoacoustic Detection of Flash-Induced Oxygen Evolution from Intact Leaves and Its Oscillations," Proceedings of National Academy of Science of USA, Vol. 85, No. 13, 1988. pp. 4725-4729. doi:10.1073/pnas.85.13.4725

[14] S. Malkin, S. K. Herbert and D. C. Fork, "Light Distribution, Transfer and Utilization in the Marine Red Alga Porphyra Perforata from Photoacoustic Energy-Storage Measurements," Biochimica et Biophysica Acta, Vol. 1016, No. 2, 1990, pp. 177-189. doi:10.1016/0005-2728(90)90056-A

[15] Z. Dubinsky, J. Feitelson and D. C. Mauzerall, "Listening to Phytoplankton: Measuring Biomass and Photosynthesis by Photoacoustics," Journal of Phycology, Vol. 34, No. 5, 1998, pp. 888-892. doi:10.1046/j.1529-8817.1998.340888.x

[16] D. C. Mauzerall, J. Feitelson and Z. Dubinsky, "Discriminating between Phytoplankton Taxa by Photoacoustics," Israel Journal of Chemistry, Vol. 38, No. 3, 1998, pp. 257-260.

[17] Y. Pinchasov, D. Kotlyarevsky, Z. Dubinsky, D. C. Mauzerall and J. Feitelson, "Photoacoustic as a Diagnostic Tool for Probing the Physiological Status of Phytoplankton," Israel Journal of Plant Sciences, Vol. 53, No. 1, 2005, pp. 1-10. doi:10.1560/4DVV-JT78-G7VW-QTFM

[18] R. R. L. Guillard and J. H. Ryther, "Studies of Marine Planktonic Diatoms. I. Cyclotella Nana Hustedt and
Detonula Confervacea (Cleve) Gran," Canadian Journal of Microbiology, Vol. 8, No. 2, 1962, pp. 229-239. doi: $10.1139 / \mathrm{m} 62-029$

[19] S. W. Jeffrey and G. F. Humphrey, "New Spectrophotometric Equation for Determining Chlorophyll A, B, $\mathrm{C}_{1}$ and $\mathrm{C}_{2}$ in Higher Plants, Algae and Natural Phytoplankton," Biochemie Physiolgie der Pflanzen, Vol. 167, 1975, pp. 194-204.

[20] P. G. Falkowski, "Light-shade Adaptation in Marine Phytoplankton Primary Productivity in the Sea," Plenum Press, New York, 1980, pp. 99-119.

[21] P. G. Falkowski, "Physiological Response of Phytoplankton to Natural Light Regimes," Journal of Plankton Research, Vol. 6, No. 2, 1984, pp. 295-307. doi:10.1093/plankt/6.2.295

[22] M. Ritz, J. C. Thomas, A. Spilar and A. L. Etienne, "Kinetics of Photoacclimation in Response to a Shift to High Light of the Red Alga Rhodella violacea Adapted to Low Irradiance," Plant Physiology, Vol. 123, No. 4, 2000, pp. 1415-1426. doi:10.1104/pp.123.4.1415

[23] H. L. MacIntyre, T. M. Kana and J. R. Geider, "The Effect of Water Motion on the Short-Term Rates of Photosynthesis by Marine Phytoplankton," Trends in Plant Science, 2000, Vol. 5, No. 1, pp. 12-17. doi:10.1016/S1360-1385(99)01504-6

[24] E. Steemann-Nielsen, "The Use of Radioactive Carbon (14C) for Measuring Organic Production in the Sea," Journal du Conseil, Perm International pour l'Exploration de la Mer, Vol. 18, 1952, pp. 117-140.

[25] T. Berner, Z. Dubinsky, F. Schanz, J. U. Grobbelaar, U. Uehlinger and P. Falkowski, "The Measurement of Primary Productivity in a High Rate Oxidation Pond," Journal of Plankton Research, Vol. 8, No. 4, 1986, pp. 659-672. doi:10.1093/plankt/8.4.659

[26] Z. Dubinsky, P. G. Falkowski, A. F. Post and U. M. van Hes, "A System for Measuring Phytoplankton Photosynthesis in a Defined Light Field with an Oxygen Electrode," Journal of Plankton Research, Vol. 9, No. 4, 1987, pp. 607-612. doi:10.1093/plankt/9.4.607

[27] P. G. Falkowski, K. Wyman, A. Ley and D. Mauzerall, "Relationship of Steady State Photosynthesis to Fluorescence in Eucaryotic Algae," Biochimica et Biophysica Acta, Vol. 849, No. 2, 1986, pp. 183-192. doi:10.1016/0005-2728(86)90024-1

[28] R. Iglesias-Prieto and R. K. Trench, "Acclimation and Adaptation to Irradiance in Symbiotic Dinoflagellates. I. Responses of the Photosynthetic Unit to Changes in Photon Flux Density," Marine Ecology Progress Series, Vol. 113, 1994, pp. 163-175. doi:10.3354/meps113163 